\title{
Association between Sleep Habits and Problems and Internet Addiction in Adolescents
}

\author{
Kentaro Kawabe ${ }^{1}$, Fumie Horiuchi ${ }^{1}$, Yasunori Oka², and Shu-ichi Ueno ${ }^{3}$ \\ ${ }^{1}$ Department of Neuropsychiatry, Ehime University Graduate School of Medicine, and Center for Child Health, Behavior and Development, \\ Ehime University Hospital, Ehime, Japan \\ ${ }^{2}$ Center for Sleep Medicine and Center for Child Health, Behavior and Development, Ehime University Hospital, Ehime, Japan \\ ${ }^{3}$ Department of Neuropsychiatry, Ehime University Graduate School of Medicine, Ehime, Japan
}

Objective This study examined the association between sleep habits and problems and Internet addiction in adolescents.

Methods Junior high school students from a local town in Japan ( $\mathrm{n}=853$; male/female, 425/428) were the subjects of this study, and were assessed for severity of Internet addiction and sleep habits and problems using the self-reported version of the Young's Internet Addiction Test (IAT) and Child and Adolescent Sleep Checklist (CASC).

Results The wake time on weekdays was not significantly different among the three groups; addicted, possibly-addicted, and non-addicted. In the addicted group, the total night sleep time was significantly shorter, and the bedtime was significantly delayed on both weekdays and weekends compared with those in the possibly-addicted and non-addicted groups. The wake time of the addicted group was significantly later than that of the other groups. The total scores of sleep problems measured by the CASC were significantly higher in the addicted and possibly-addicted groups than in the non-addicted group.

Conclusion Internet addiction is strongly associated with sleep habits and problems in adolescents. These findings suggest that internet addiction should be considered while examining adolescent lifestyle.

Psychiatry Investig 2019;16(8):581-587

Key Words Adolescent, Internet addiction, Internet-use disorder, Sleep habits, Smartphone.

\section{INTRODUCTION}

The Internet has provided a new communication medium that enables access to unlimited information resources across numerous topics. In the past decades, Internet use has greatly increased worldwide. The Internet penetration rate in Japan is $83.0 \%$ across all age groups according to the Ministry of Internal Affairs and Communications survey in 2016. Among 13-49-year-olds, the Internet penetration rate has reached $95 \%,{ }^{1}$ demonstrating that the Internet has become an essential aspect of our life. However, heavy Internet use has been associated with potentially detrimental side effects. Generally, adolescents spend more time on the Internet than adults, ${ }^{2}$

Received: August 2, 2018 Revised: March 18, 2019

Accepted: March 21, 2019

$\triangle$ Correspondence: Fumie Horiuchi, MD, PhD

Department of Neuropsychiatry, Ehime University Graduate School of Medicine, Center for Child Health, Behavior and Development, Ehime University Hospital, Shitsukawa, Toon city, Ehime 7910295, Japan

Tel: +81-89-960-5315, Fax: +81-89-960-5317, E-mail: matusfu@m.ehime-u.ac.jp

(c) This is an Open Access article distributed under the terms of the Creative Commons Attribution Non-Commercial License (https://creativecommons.org/licenses/by$\mathrm{nc} / 4.0$ ) which permits unrestricted non-commercial use, distribution, and reproduction in any medium, provided the original work is properly cited. predisposing themselves to Internet addiction (IA). IA criteria comprise the following four components: 1) excessive Internet use, often associated with a loss of sense of time or a neglect of basic drives, 2) withdrawal, including feelings of anger, depression, and tension when the Internet is inaccessible, 3) lack of tolerance, especially regarding the need for better computer equipment, amount of software, or more hours of use, and 4) negative repercussions, including arguments, lying, poor achievement, social isolation, and fatigue. ${ }^{3}$ Serious problems associated with IA among adolescents include refusal to attend school, negative influence on family relationships, and mental health problems, such as loneliness, low self-esteem, less life satisfaction, poor academic performance, insufficient sleep, anxiety, and depression..$^{4-8}$ In addition, close peers are associated in the development of IA. ${ }^{9}$ IA and sleep problems are common among children and adolescents, and are a main concern for parents. Students who spend more time watching television, playing computer games, and using the Internet eventually spend less time sleeping and experience higher levels of tiredness. ${ }^{10}$ The use of computer games and mobile phones in the bedroom is re- 
lated to poor sleep habits, learning, and memory in children. ${ }^{11-13}$ Stegar reported that Japanese high school students often study until late at night and sacrifice their sleep, and then nap during the afternoon, which is widely accepted. ${ }^{14}$ Nightmares and sleep paralysis were also found to be associated with daytime naps according to a cross-sectional survey of junior and senior high school students in Japan. ${ }^{15}$ We previously reported a strong correlation between IA and mental health problems, such as suicidal depression, and the accessibility to smartphones in junior high school students. ${ }^{16} \mathrm{Re}-$ cently, the importance of sleep for a high quality and healthy life both physically and psychologically has been reevaluated in Japan. ${ }^{17}$ A previous study on Japanese adult psychiatric patients revealed that problematic internet users had significantly higher scores for sleep problems. ${ }^{18}$ However, there are no reports on the association between sleep habits and problems and IA among Japanese adolescents.

We hypothesized that sleep habits and problems in adolescents are significantly associated with IA. Thus, the aim of this study was to examine the association of IA with sleep habits/problems in adolescents.

\section{METHODS}

\section{Participants}

In November 2014, participants were recruited from three junior high schools in Masaki town in Ehime Prefecture, which is a rural area close to the sea in western Japan. The average income level in Ehime prefecture is the same as the national average, and the overall unemployment rate (for those aged $\geq 15$ years) in Ehime in 2017 (2.3\%) was lower than the national rate $(2.8 \%)$. In Japan, public schools generally start at 8:30 a.m. and finish at 4:00 p.m. on weekdays. The population of the surveyed area, Masaki town, is 31,000, and it is increasing. There are 3 public junior high schools in the survey area. All junior high school students in the area ( $n=874$; age, $12-15$ years) were recruited in the study. Two students did not participate in the study, and 19 students did not respond to at least one questionnaire; therefore, we excluded 21 students. In total, 853 participants (males, $n=425$; females, $n=428$ ) were included in the analysis (proper response rate, $97.6 \%)$.

\section{Materials}

Participants responded to the Young's Internet Addiction Test (IAT), Child and Adolescent Sleep Checklist (CASC), and their usage of or exposure to electronic devices.

\section{Measurement of IA}

The 20 items that comprise the IAT are scored on a scale ranging from 1 to 5 (total scores ranging from 20 to 100), with higher scores reflecting a greater tendency toward addiction. ${ }^{19}$ Three types of Internet users were identified in accordance with the original scheme of Young. ${ }^{20}$ Respondents with IAT scores $>70$ were classified as "addicted," and most likely had encountered significant life problems due to excessive Internet use. Respondents with IAT scores between 40 and 69 were classified as "possibly-addicted," and those scoring <39 were considered "non-addicted" and were most likely average Internet users, with only some problems in controlling their Internet use. The cut-off points were determined by referring to previous studies conducted in Japan. ${ }^{21,22}$ Although the Japanese version of the IAT has not been validated, the originally validated IAT has been used globally. In the present study sample ( $\mathrm{n}=853)$, Cronbach's a coefficient was 0.929 .

\section{Measurement of sleep habits and problems}

Sleep habits and problems among students were assessed using the high school students' version of the CASC. ${ }^{23}$ It was designed to identify sleep habits and screen for sleep problems among preschoolers, elementary school children, and high school students. Currently available language options are English and Japanese. It consists of 12 questions regarding sleep habits, and a 24 -item checklist that addresses sleeprelated problems. Usual bedtime (BT), wake time (WT), and total night sleep time (TST) on weekdays and weekends over the previous week were obtained from the self-reported version of the CASC. Individual weekend catch-up sleep duration was calculated as the weekend sleep duration minus the weekday sleep duration. Regarding the 24 items, participants were asked to respond using a 4-point frequency scale in which 0 indicated "never and unknown," 1 indicated "occasionally" (0-1 day per week), 2 indicated "sometimes" (2-4 days per week), and 3 indicated "always" (5-7 days per week). The 24 items that comprise sleep disturbance were scored on a scale ranging from 0 to 3 (total score ranging from 0 to 72 ). CASC scores were subdivided into three categories: pre-sleep domain, Q1-6 (6 questions); nighttime domain, Q7-19 (13 questions); and daytime domain, Q20-24 (5 questions). In the present study sample ( $\mathrm{n}=853)$, Cronbach's a coefficient was 0.786 .

\section{Measurement of usage of and exposure to electronic devices}

Participants were asked to fill out a questionnaire on their usage of electronic devices such as televisions, radios, cell phones, smartphones, laptop computers, tablet computers, console games, including computer games, and portable games. Participants were also asked to respond whether electronic devices were freely accessible to them or placed in their own room with 'yes' or 'no.' 


\section{Sample size calculation}

The sample size was calculated based on a multiple logistic regression model using $\mathrm{G}^{*}$ power 3.1.9.2 software. ${ }^{24}$ A small size effect of 0.02 , a significance level of alpha $=0.05$, and a statistical power of $1-\beta=0.95$ were considered. The necessary sample size is fulfilled in this study.

\section{Ethical considerations}

This study was approved by the Institutional Review Board of the Ehime University Graduate School of Medicine, with the cooperation of the School Board of Masaki town (IRB No. 1410021). The study protocol was explained to the junior high school teachers, and parents were informed of this study via a letter and were asked to contact the school for further clarification. In November 2014, homeroom teachers explained the purpose of the study to their students, who then filled the questionnaires during class using a self-reporting format. It took about 20 minutes were required which students to filled out these questionnaires. The questionnaires are anonymous, so the individual result is uninformed to each student. Students were informed that they could freely determine the entry of this study and reassured that this would not affect their school life in any ways. Written informed consent was obtained from each student.

\section{Data analysis}

Continuous variables were expressed as mean \pm SD. Categorical variables were expressed as numbers and percentages. For categorical variables, chi-square tests were employed to compare the number of participants in the non-addicted, possibly-addicted, and addicted groups, complemented by adjusted residual analysis. In addition, a one-way analysis of variance (ANOVA) was conducted to compare CASC scores among the three IAT groups with post hoc analysis using the Bonferroni correction. Next, we defined the participants with IAT scores of $>40$, who were included in the addicted and possibly-addicted groups with IA. Logistic regression models were used to assess the independent associations between IA and sleep problems evaluated by CASC. We employed a multivariate model and backward stepwise regression. All tests were two-sided and assumed a 5\% significance level. All data were analyzed using the statistical software SPSS Statistics (IBM Corp., Armonk, NY, USA) for Windows, version 23.0.

\section{RESULTS}

The mean age of the students was $13.6 \pm 0.9$ years (male, $13.6 \pm 0.9$ years; female, $13.5 \pm 1.0$ years). Of the 853 students (male/female, 425/428), 279 (12-13 years old; male/female, 126/153) were first grade, 271 (13-14 years old; male/female, 144/127) were second grade, and 303 (14-15 years old; male/ female, 155/148) were third grade students. Based on the total IAT scores, $2.0 \%$ (male, $2.1 \%$; female, $1.9 \%$ ) and $21.7 \%$ (male, 19.8\%; female, 23.6\%) of adolescents were classified as addicted and possibly-addicted, respectively (Table 1). As a result, the prevalence of IA, including both addicted and possibly-addicted adolescents, was 23.7\% (male, 21.9\%; female, 25.5\%). Table 1 depicts the demographic features of the participants according to their IA-status. No significant gender differences were found. Regarding grade, first grade participants classified as non-addicted had significantly higher prevalence rates than third grade participants, and third grade participants classified as possibly-addicted had significantly higher prevalence rates than first grade participants.

\section{The sleep habits/problems of students with IA}

As shown in Table 2, the TST in the addicted group on weekdays was significantly shorter than that in other groups, and the TST in the possibly-addicted group was significantly

Table 1. Demographics of Internet use according to status of Internet addiction

\begin{tabular}{lccc}
\hline \multirow{2}{*}{ Demographics } & \multicolumn{3}{c}{ IA-status } \\
\cline { 2 - 4 } Total, N (\%) & Non-addicted (IAT<40) & Possibly-addicted (IAT: 40-69) & Addicted (IAT>70) \\
Sex, N (\%) & $651(76.3)$ & $185(21.7)$ & $17(2.0)$ \\
$\quad$ Male & $332(78.1)$ & & $9(2.1)$ \\
$\quad$ Female & $319(74.5)$ & $84(19.8)$ & $8(1.9)$ \\
Junior high school grade, N (\%) & & $101(23.6)$ & $5(1.8)$ \\
$\quad$ First & $238(85.3)$ & $36(12.9)$ & $6(2.2)$ \\
$\quad$ Second & $201(74.2)$ & $64(23.6)$ & $6(2.0)$ \\
$\quad$ Third & $212(70.0)$ & $85(28.0)$ & $81.1 \pm 8.8$ \\
IAT score & $26.2 \pm 5.7$ & $49.8 \pm 8.3$ & $<0.001^{*}$ \\
\hline
\end{tabular}

Chi-square test; ${ }^{*} \mathrm{p}<0.01$. IAT: Young's Internet Addiction Test 
Table 2. Analysis of variance for Child and Adolescent Sleep Checklist (CASC) subscale scores and sleep habits/problems in the three groups

\begin{tabular}{|c|c|c|c|c|c|c|}
\hline Sleep habits & $\begin{array}{c}\text { Total } \\
\mathrm{N}=853\end{array}$ & $\begin{array}{c}\text { Non-addicted } \\
\mathrm{N}=651(\mathrm{~A})\end{array}$ & $\begin{array}{c}\text { Possibly-addicted } \\
\text { N=185 (B) }\end{array}$ & $\begin{array}{l}\text { Addicted } \\
\mathrm{N}=17(\mathrm{C})\end{array}$ & $\mathrm{F}$ & $\mathrm{p}^{*}$ \\
\hline \multicolumn{7}{|l|}{ Total night sleep time } \\
\hline Weekdays, minutes & $422.1 \pm 77.4$ & $431.2 \pm 72.4$ & $397.9 \pm 80.6$ & $323.0 \pm 113.2$ & 27.0 & $<0.001^{\dagger, \neq, \S}$ \\
\hline Weekends, minutes & $482.4 \pm 96.4$ & $487.8 \pm 91.6$ & $467.5 \pm 101.8$ & $428.3 \pm 174.5$ & 5.6 & $0.004^{\dagger}$ \\
\hline \multicolumn{7}{|l|}{ Wake time } \\
\hline Weekdays & $6: 24 \pm 0: 35$ & $6: 23 \pm 0: 35$ & $6: 25 \pm 0: 33$ & $6: 40 \pm 0: 37$ & 2.0 & 0.140 \\
\hline Weekends & $7: 48 \pm 1: 26$ & $7: 41 \pm 1: 19$ & $8: 04 \pm 1: 41$ & $9: 13 \pm 2: 24$ & 11.1 & $<0.001^{\dagger, \ddagger, \S}$ \\
\hline \multicolumn{7}{|l|}{ Bedtime } \\
\hline Weekdays & $23: 09 \pm 1: 00$ & $23: 02 \pm 0: 54$ & $23: 25 \pm 1: 10$ & $24: 32 \pm 1: 17$ & 27.8 & $<0.001^{\dagger, \pm, \S}$ \\
\hline Weekends & $23: 26 \pm 1: 11$ & $23: 16 \pm 1: 02$ & $23: 51 \pm 1: 20$ & $25: 10 \pm 2: 12$ & 37.5 & $<0.001^{\dagger, \ddagger \S}$ \\
\hline Weekend catch-up, minutes & $60.7 \pm 91.0$ & $56.7 \pm 84.9$ & $71.4 \pm 105.2$ & $105.3 \pm 135.5$ & 3.7 & 0.025 \\
\hline \multicolumn{7}{|l|}{ CASC } \\
\hline Total & $14.9 \pm 7.4$ & $13.3 \pm 6.6$ & $19.3 \pm 6.7$ & $27.8 \pm 12.1$ & 87.0 & $<0.001^{\dagger, \ddagger, \S}$ \\
\hline Pre-sleep & $4.9 \pm 2.8$ & $4.4 \pm 2.6$ & $6.4 \pm 2.5$ & $8.8 \pm 3.3$ & 66.2 & $<0.001^{\dagger, \ddagger, \S}$ \\
\hline Nighttime & $4.8 \pm 3.8$ & $4.3 \pm 3.4$ & $6.1 \pm 3.8$ & $9.9 \pm 9.2$ & 34.2 & $<0.001^{\dagger, \ddagger, \S}$ \\
\hline Daytime & $5.2 \pm 2.9$ & $4.7 \pm 2.7$ & $6.7 \pm 2.9$ & $9.1 \pm 2.7$ & 58.3 & $<0.001^{\dagger, \ddagger \S \S}$ \\
\hline
\end{tabular}

*ANOVA, $\mathrm{p}<0.05$, the score of $\mathrm{A}$ is significantly lower than that of $\mathrm{B}$, the score of $\mathrm{B}$ is significantly lower than that of $\mathrm{C}$, sthe score of $\mathrm{A}$ is significantly lower than that of $\mathrm{C}$

shorter than that in the non-addicted group. In addition, the TST on weekends was significantly shorter in the possiblyaddicted group than that in the non-addicted group. WT on weekdays was not different among the three groups; however, it was significantly later in the addicted group than in the other two groups on weekends and in the possibly-addicted group than in the non-addicted group. BT in the addicted group on both weekdays and weekends was significantly delayed compared with the other groups; it was also significantly delayed in the possibly-addicted group compared with the non-addicted group. Weekend catch-up sleep significantly differed among three groups; however, post hoc tests did not reveal significant differences among the groups. The total scores and each subscale of sleep problems measured by CASC were significantly different among the three groups.

\section{Factors that contribute to IA}

As shown in Table 3, univariate logistic regression analyses revealed that 20 variables were significantly associated with IA. Using a multivariate model with IA as the dependent variable, we found that the following items had significantly high odds ratios (ORs): pre-sleep domain: "I play video games, surf the Internet, or send texts one hour or less before going to bed" [crude OR=2.56 and 95\% confidence interval $(\mathrm{CI})=$ 2.06-3.17; adjusted $\mathrm{OR}=2.51$ and $95 \% \mathrm{CI}=1.19-3.74]$ and "I avoid going to bed even though it is time to go to sleep" (crude $\mathrm{OR}=2.56$ and $95 \% \mathrm{CI}=1.51-2.10$; adjusted $\mathrm{OR}=1.31$ and 95\% CI=1.08-1.59); nighttime domain: "I cry out in my sleep and wake up during the night." (crude $\mathrm{OR}=2.51$ and 95\% $\mathrm{CI}=1.61-3.90$; adjusted $\mathrm{OR}=2.11$ and $95 \% \mathrm{CI}=1.19-3.74)$ and "The amount of sleep I get varies each night." (crude $\mathrm{OR}=1.83$ and $95 \% \mathrm{CI}=1.52-2.19$; adjusted $\mathrm{OR}=1.37$ and $95 \% \mathrm{CI}=1.10$ 1.69); daytime domain: "I feel tired or groggy when I wake up in the morning." (crude $\mathrm{OR}=1.83$ and $95 \% \mathrm{CI}=1.54-2.17$; adjusted $\mathrm{OR}=1.38$ and $95 \% \mathrm{CI}=1.13-1.69$ ) and "I get sleepy during class." (crude $\mathrm{OR}=2.02$ and $95 \% \mathrm{CI}=1.66-2.45$; adjusted $\mathrm{OR}=1.43$ and $95 \% \mathrm{CI}=1.13-1.81$ ).

\section{DISCUSSION}

This study focused on the association between sleep habits/problems and IA in junior high school students. Based on our results, several sleep habits, including TST, WT, BT, and subscales of sleep problems measured by CASC were significantly different among the addicted, possibly-addicted, and non-addicted groups. A previous study by Chen and $\mathrm{Gau}^{25}$ is a 1-year longitudinal survey to evaluate the bidirectional relationships between sleep problems and IA among children and adolescents. Their study findings showed that IA is associated with decreased sleep duration during the night- time and dyssomnias sequentially predicted IA, and IA sequentially predicted disturbed circadian rhythm. However, their study did not indicate the association between IA and qualities of sleep problems, which are insomnia, periodic leg move- 
Table 3. Association of Internet addiction (IA) with factors on the Child and Adolescent Sleep Checklist (CASC)

\begin{tabular}{|c|c|c|c|}
\hline & \multirow{2}{*}{ Checklist } & \multicolumn{2}{|c|}{ Adjusted } \\
\hline & & OR & $95 \% \mathrm{CI}$ \\
\hline \multirow{6}{*}{$\begin{array}{c}\text { Pre-sleep } \\
\text { domain }\end{array}$} & I drink a caffeinated beverage three hours or less before going to bed. & & \\
\hline & I play video games, surf the Internet, or send texts one hour or less before going to bed. & $2.16^{\dagger}$ & $1.73-2.71$ \\
\hline & I avoid going to bed even though it is time to go to sleep. & $1.31^{\dagger}$ & $1.08-1.59$ \\
\hline & I feel anxious or afraid when it is time to go to sleep. & & \\
\hline & I have trouble falling asleep when I am by myself. & & \\
\hline & Before I fall asleep, my legs feel uncomfortable like I cannot hold them still. & & \\
\hline \multirow{13}{*}{$\begin{array}{l}\text { Nighttime } \\
\text { domain }\end{array}$} & I snore. & & \\
\hline & My breath sounds as if it is getting caught in my throat. & & \\
\hline & I stop breathing while I sleep. & & \\
\hline & I toss and turn, or change positions often while I sleep. & & \\
\hline & I talk in my sleep. & & \\
\hline & I cry out in my sleep and wake up during the night. & $2.11^{*}$ & $1.19-3.74$ \\
\hline & I have scary dreams or cry out during nightmares. & & \\
\hline & I sleepwalk. & & \\
\hline & My legs twitch while I sleep. & & \\
\hline & I urinate in my sleep. & & \\
\hline & I grind my teeth while I sleep. & & \\
\hline & I sweat excessively while I sleep. & & \\
\hline & The amount of sleep I get varies each night. & $1.37^{\dagger}$ & $1.10-1.69$ \\
\hline \multirow{5}{*}{$\begin{array}{r}\text { Daytime } \\
\text { domain }\end{array}$} & I feel tired or groggy when I wake up in the morning. & $1.38^{\dagger}$ & $1.13-1.69$ \\
\hline & I skip breakfast. & & \\
\hline & I get sleepy during class. & $1.43^{\dagger}$ & $1.13-1.81$ \\
\hline & I fall asleep during class. & & \\
\hline & I fall asleep if I sit still. (i.e., watching TV, riding in the car) & & \\
\hline
\end{tabular}

${ }^{*} \mathrm{p}<0.05,{ }^{\dagger} \mathrm{p}<0.01$. OR: odds ratio, CI: confidence interval

ments, sleep terrors, sleepwalking, sleep talking, nightmares, bruxism, snoring and sleep apnea. Although our current study was performed by different methodologic and different assessment tools, it is significantly shorter total sleep duration in children with IA. Furthermore, our study indicated that the amount of sleep time varies considerably from day to day in children with IA. To the best of our knowledge, the present study is the first to demonstrate an association between sleep habits/problems and IA among Japanese junior high school students.

\section{The total sleep duration by children with IA}

According to the present study, the average TST on weekdays was $<7 \mathrm{~h}$ in both the addicted and possibly-addicted groups. According to Wolfson and Carskadon, ${ }^{26}$ with the onset of adolescence, teenagers require $9.2 \mathrm{~h}$ of sleep per day. Silva et al. ${ }^{27}$ reported that children who slept for $<7.5 \mathrm{~h} /$ night had a high risk of being overweight or obese, anxious or depressed, and having learning problems compared with those who slept for $\geq 9 \mathrm{~h} /$ night. The TST was comparatively shorter in the possibly-addicted and addicted groups. Several studies have found that sleep problems, including short sleep duration, can seriously affect school performance and cause depression in adolescents. ${ }^{28,29}$ Thus, we need to carefully observe sleep habits and total sleep duration, and communicate the importance of sleep to adolescents with IA.

\section{Weekend catch-up sleep by children with IA}

The WT on weekends in the addicted and possibly-addicted groups was significantly later than that in the non-addicted group. Shorter weekday sleep duration and longer weekend oversleeping is associated with developing IA. Increased weekend catch-up sleep by adolescents as an indicator of insufficient weekday sleep was associated with poor performance on objective attention tasks. ${ }^{30}$ In addition, weekend oversleep was found to be associated with suicidal tendencies, but independent of depression, daytime sleepiness, snoring, and insomnia among adolescents. ${ }^{31}$ Longer weekend catch-up sleep should be considered in terms of prevention of IA. 


\section{Details regarding sleep problems in children with IA}

The addicted and possibly-addicted groups exhibited more sleep problems than the non-addicted group across all subscales of the CASC. Our study also revealed the details of sleep problems among students with IA using the logistic regression model. The students categorized as having IA had sleep problems in all the three domains (pre-sleep, nighttime, and daytime). The most notable subscale item with the highest OR was "I play video games, surf the Internet, or send texts one hour or less before going to bed," followed by "I cry out in my sleep and wake up during the night." There were 43 participants who did not respond "0" to "I cry out in my sleep and wake up during the night." In this questionnaire, "cry out during the night" indicates sleep terrors, which are defined as an event that produces a partially aroused state from deep sleep. Sleep terrors are a part of a larger group of parasomnias and are characterized by marked autonomic nervous system activation, where tachycardia, tachypnea, tremulousness, mydriasis, and sweating are often present. ${ }^{32}$ The prevalence of sleep terrors has not been reported in Japan. The prevalence of sleep terrors was $5.1 \%(43 / 853)$ in this study. There was also low prevalence $(3.65 \%)$ of sleep terrors in a normal school-age population according to Belgian study. ${ }^{33}$ To date, there has been very little research on the relationship between sleep terrors and IA. A past study by Choi et al. ${ }^{34}$ reported an association between IA and sleep problems, but no association between IA and sleep terrors. ${ }^{34}$ Factors that may increase the likelihood of sleep terror events in susceptible individuals include acute stress associated with sleep deprivation. High stimulation prior to bedtime may be one of the causal factors for sleep terrors. Based on this study, sleep terrors may be partially associated with IA in adolescents.

The next notable subscale item was "I get sleepy during class." According to Choi et al., ${ }^{34}$ Internet overuse is highly associated with excessive daytime sleepiness in adolescents, and IA causes some sleep behavioral problems in many situations, including at school or home. The American Academy of Pediatrics (AAP) has expressed concerns about the amount of time that children and teenagers spend watching media and about some of the content they view. ${ }^{35}$ AAP recommends keeping the TV set and Internet-connected electronic devices out of the bedroom, and enforcing a mealtime and bedtime "curfew" on the use of such equipment. In addition, it recommends monitoring the media or websites that children access, supervising children while they watch TV, movies, and videos, and use this as a way of discussing important family values. Taken together, the findings of the present study suggest that IA contributes to sleep problems, and that it is important to prevent IA in order to attenuate its detrimental effects on the sleep habits of adolescents.

\section{Limitations}

The present study has several limitations. First, our assessment relied on self-reports. We did not control for confounding factors such as economic status and academic performance. IA is significantly related to poor academic attainment with the previous study. ${ }^{36}$ In addition, it has been reported that IA may have one or more psychiatric comorbidities. ${ }^{37}$ However, we did not assess any psychiatric symptoms related to depression, anxiety disorder, obsessive compulsive disorder, attention deficit disorder, or neurodevelopmental disorder in this study. Therefore, our findings may underestimate the seriousness of IA and sleep problems. Second, as the present study was cross-sectional, it cannot demonstrate cause and effect relationships, different from longitudinal studies. Third, study participants were recruited from public junior high schools in suburban areas. Although the recruitment area was suburban and relatively intermediate in terms of socioeconomics, our findings may not be generalized for the entire Japanese adolescent population. Fourth, the IAT was developed approximately 20 years ago when internet access and proliferation of multimedia devices was limited compared with now.

In conclusion, this study revealed a strong association between IA and sleep habits/problems among Japanese adolescents. We suggest that further studies be undertaken to corroborate these findings, and to develop strategies to moderate the use of the Internet and improve the quantity and quality of sleep in this population.

\section{Conflicts of Interest}

The authors have no potential conflicts of interest to disclose.

\section{Author Contributions}

Kentaro Kawabe designed the study, managed data collection, conducted statistical analysis and prepared the initial manuscript. Fumie Horiuchi contributed to study design, data collection, statistical analysis, and revision of the manuscript critically for important intellectual content. Both Yasunori Oka and Shu-ichi Ueno provided critical comment which enhanced significantly the final draft. All authors approved the final version.

\section{ORCID iDs}

Fumie Horiuchi https://orcid.org/0000-0002-0833-0173

Kentaro Kawabe https://orcid.org/0000-0002-6569-7828

\section{REFERENCES}

1. White Paper Information and Communications in Japan. Tokyo: Ministry of Internal Affairs and Communications; 2016.

2. Kraut R, Kiesler S, Boneva B, Cummings J, Helgeson V, Crawford A. Internet paradox revisited. J Soc Issues 2002;58:49-74.

3. Spada MM. An overview of problematic internet use. Addict Behav 2014;39:3-6.

4. Kaltiala-Heino R, Lintonen T, Rimpelä A. Internet addiction? Potentially problematic use of the internet in a population of $12-18$ year-old adolescents. Addict Res Theory 2004;12:89-96.

5. Kim K, Ryu E, Chon MY, Yeun EJ, Choi SY, Seo JS, et al. Internet ad- 
diction in Korean adolescents and its relation to depression and suicidal ideation: a questionnaire survey. Int J Nurs Stud 2006;43:185-192.

6. Ko CH, Yen JY, Yen CF, Lin HC, Yang MJ. Factors predictive for incidence and remission of internet addiction in young adolescents: a prospective study. CyberPsychol Behav 2007;10:545-551.

7. Wainer J, Dwyer T, Dutra RS, Covic A, Magalhães VB, Ferreira LRR, et al. Too much computer and Internet use is bad for your grades, especially if you are young and poor: results from the 2001 Brazilian SAEB. Comput Educ 2008;51:1417-1429.

8. Lin F, Zhou Y, Du Y, Qin L, Zhao Z, Xu J, et al. Abnormal white matter integrity in adolescents with internet addiction disorder: a tract-based spatial statistics study. PLoS One 2012;7:e30253.

9. Zhou N, Fang XY. Beyond peer contagion: unique and interactive effects of multiple peer influences on Internet addiction among Chinese adolescents. Comput Human Behav 2015;50:231-238.

10. Van den Bulck J. Television viewing, computer game playing, and Internet use and self-reported time to bed and time out of bed in secondary-school children. Sleep 2004;27:101-104.

11. Dworak M, Schierl T, Bruns T, Strüder HK. Impact of singular excessive computer game and television exposure on sleep patterns and memory performance of school-aged children. Pediatrics 2007;120: 978-985.

12. Shochat T, Flint-Bretler O, Tzischinsky O. Sleep patterns, electronic media exposure and daytime sleep-related behaviours among Israeli adolescents. Acta Paediatr 2010;99:1396-1400.

13. Brunborg GS, Mentzoni RA, Molde H, Myrseth H, Skouverøe KJM, Bjorvatn B, et al. The relationship between media use in the bedroom, sleep habits and symptoms of insomnia. J Sleep Res 2011:20;569-575.

14. Steger B. Sleeping through class to success: Japanese notions of time and diligence. Time Soc 2006;15:197-214.

15. Munezawa T, Kaneita Y, Osaki Y, Kanda H, Ohtsu T, Suzuki H, et al. Nightmare and sleep paralysis among Japanese adolescents: a nationwide representative survey. Sleep Med 2011;12:56-64.

16. Kawabe K, Horiuchi F, Ochi M, Oka Y, Ueno SI. Internet addiction: prevalence and relation with mental states in adolescents. Psychiatry Clin Neurosci 2016;70:405-412.

17. Miyazaki S, Sato S, Kitamura T, Morikuni I, Tsuji N, Honda K, et al. Sleep education and awareness-raising activities in Japan. Sleep Biol Rhythms 2016;14:3-9.

18. de Vries HT, Nakamae T, Fukui K, Denys D, Narumoto J. Problematic internet use and psychiatric co-morbidity in a population of Japanese adult psychiatric patients. BMC Psychiatry 2018;18:9.

19. Young KS. Psychology of computer use: XL. Addictive use of the Internet: a case that breaks the stereotype. Psychol Rep 1996;79:899-902.

20. Young KS. Caught in the Net: How to Recognize the Signs of Internet Addiction--and a Winning Strategy for Recovery. New York City: John Wiley and Sons; 1998.

21. Tateno M, Teo AR, Shirasaka T, Tayama M, Watabe M, Kato TA. Internet addiction and self-evaluated attention-deficit hyperactivity dis- order traits among Japanese college students. Psychiatry Clin Neurosci 2016;70:567-572.

22. So R, Makino K, Fujiwara M, Hirota T, Ohcho K, Ikeda S, et al. The prevalence of internet addiction among a Japanese adolescent psychiatric clinic sample with autism spectrum disorder and/or attentiondeficit hyperactivity disorder: a cross-sectional study. J Autism Dev Disord 2017;47:2217-2224.

23. Oka Y, Horiuchi F, Tanigawa T, Suzuki S, Kondo F, Sakurai S, et al. Development of a new sleep screening questionnaire: Child and Adolescent Sleep Checklist (CASC). Japanese. J Sleep Med 2009;3:404-408.

24. Faul F, Erdfelder E, Buchner A, Lang AG. Statistical power analyses using $\mathrm{G}^{*}$ Power 3.1: tests for correlation and regression analyses. Behav Res Methods 2009;41:1149-1160.

25. Chen YI, Gau SSF. Sleep problems and internet addiction among children and adolescents: a longitudinal study. J Sleep Res 2016;25:458-465.

26. Wolfson AR, Carskadon MA. Understanding adolescent's sleep patterns and school performance: a critical appraisal. Sleep Med Rev 2003;7:491-506.

27. Silva GE, Goodwin JL, Parthasarathy S, Sherrill DL, Vana KD, Drescher AA, et al. Longitudinal association between short sleep, body weight, and emotional and learning problems in Hispanic and Caucasian children. Sleep 2011;34:1197-1205.

28. Curcio G, Ferrara M, De Gennaro L. Sleep loss, learning capacity and academic performance. Sleep Med Rev 2006;10:323-337.

29. Lovato N, Gradisar M. A meta-analysis and model of the relationship between sleep and depression in adolescents: recommendations for future research and clinical practice. Sleep Med Rev 2014;18:521-529.

30. Kim SJ, Lee YJ, Cho SJ, Cho IH, Lim W, Lim W. Relationship between weekend catch-up sleep and poor performance on attention tasks in Korean adolescents. Arch Pediatr Adolesc Med 2011;165:806-812.

31. Lee YJ, Cho SJ, Cho IH, Kim SJ. Insufficient sleep and suicidality in adolescents. Sleep 2012;35:455-460.

32. Mason TB, Pack AI. Sleep terrors in childhood. J Pediatr 2005;147: 388-392.

33. Spruyt K, O'brien LM, Cluydts R, Verleye GB, Ferri R. Odds, prevalence and predictors of sleep problems in school-age normal children. J Sleep Res 2005;14:163-176.

34. Choi K, Son H, Park M, Han J, Kim K, Lee B, et al. Internet overuse and excessive daytime sleepiness in adolescents. Psychiatry Clin Neurosci 2009;63:455-462.

35. Strasburger VC, Hogan MJ, Mulligan DA, Ameenuddin N, Christakis DA, Cross C, et al. Children, adolescents, and the media. Pediatrics 2013;5:958-961.

36. Hawi NS, Samaha M, Griffiths MD. Internet gaming disorder in Lebanon: relationships with age, sleep habits, and academic achievement. J Behav Addict 2018;7:70-78.

37. Ko CH, Yen JY, Yen CF, Chen CS, Chen CC. The association between Internet addiction and psychiatric disorder: a review of the literature. Eur Psychiatry 2012;27:1-8. 